Supporting Information

Strategy for Activating the Room Temperature Phosphorescence of Carbon Dots in Aqueous Environment

Yifang Gao, $\ddagger$ Huilin Zhang, $\ddagger$ Yuan Jiao, Wenjing Lu, Yang Liu, Hui Han, Xiaojuan Gong, Shaomin Shuang and Chuan Dong* 


\section{Synthesis of CDs-1}

Diethylenetriaminepentaacetic acid was sufficiently mixed via sonication with $30 \mathrm{ml}$ of DDI water solution, and then the mixture was heated to $200{ }^{\circ} \mathrm{C}$ for $3 \mathrm{~h}$ by hydrothermal treatment. The remaining steps are the same as those for synthesizing M-CDs.

Synthesis of CDs-2

Glucose and L- Aspartic Acid were sufficiently mixed via sonication with $3 \mathrm{ml}$ of aqueous sodium hydroxide solution, and then the mixture was heated to $150^{\circ} \mathrm{C}$ by an oil bath. The remaining steps are the same as those for synthesizing M-CDs.

Synthesis of CDs-3

Sodium polyacrylate and urea was sufficiently mixed via sonication with $30 \mathrm{ml}$ of DDI water solution, and then the mixture was heated to $200{ }^{\circ} \mathrm{C}$ for $3 \mathrm{~h}$ by hydrothermal treatment. The remaining steps are the same as those for synthesizing M-CDs.

Determination of Quantum yields

Quantum yields of the CDs was concluded by absolute method using integrating sphere.

1. Measurement of the Base/Standard (solvent • base reflector): Amounts of excitation light and

background fluorescence are measured;

2. Sample Measurement: Amounts of reflected light and fluorescence and measured;

3. Calculation: The quantum yield is calculated based on the absorbed amount and fluorescence amount.

Specifically, aluminum oxide was voted as highly reflecting white material. The QY of a sample was determined by the following equation:

$$
\phi=\phi_{\mathrm{d}}-\left(1-\mathrm{A}_{\mathrm{d}}\right) \phi_{\mathrm{i}}
$$

where $\phi_{d}, A_{d}$ and $\phi_{i}$ represent quantum yield by direct excitation, absorption rate by direct excitation and quantum yield by indirect excitation, respectively.

Table S1. Elemental analysis of the as-synthesised CDs.

\begin{tabular}{ccccc}
\hline Sample name & \multicolumn{4}{c}{ Elemental content (\%) } \\
\cline { 2 - 5 } & $\mathrm{C}$ & $\mathrm{H}$ & $\mathrm{N}$ & $\mathrm{O}$ (Calculated) \\
$\mathrm{CDs}$ & 26.95 & 5.51 & 13.30 & 54.24 \\
\hline
\end{tabular}

Table S2. Lifetime calculations from the time-resolved decay profiles of CDs and M-CDs.

\begin{tabular}{cccccc}
\hline Sample & $\tau 1(\mathrm{~ms})$ & $\begin{array}{c}\text { Percentage } \\
(\%)\end{array}$ & $\tau 2(\mathrm{~ms})$ & $\begin{array}{c}\text { Percentage } \\
(\%)\end{array}$ & Ave. $\tau(\mathrm{ms})$ \\
\hline CDs & 94.10 & 23.18 & 581.60 & 76.82 & 447 \\
M-CDs & 117.78 & 30.35 & 901.76 & 69.65 & 664 \\
\hline
\end{tabular}

Table S3. Comparison of the Quantum yields between the M-CDs and other CDs.

\begin{tabular}{ccc}
\hline Materials & $\begin{array}{c}\text { Quantum } \\
\text { yields }\end{array}$ & Refs. \\
\hline CDs & $5.99 \%$ & 1 \\
\hline
\end{tabular}




\begin{tabular}{ccc}
\hline NCDs & $7 \%$ & 2 \\
N-doped CQDs & $11 \%$ & 3 \\
F-CDs & $21.82 \%$ & 4 \\
CDs & $3.53 \%$ & 5 \\
a-CDs/BA & $8.7 \%$ & 6 \\
M-CDs & $25 \%$ & This work \\
\hline
\end{tabular}
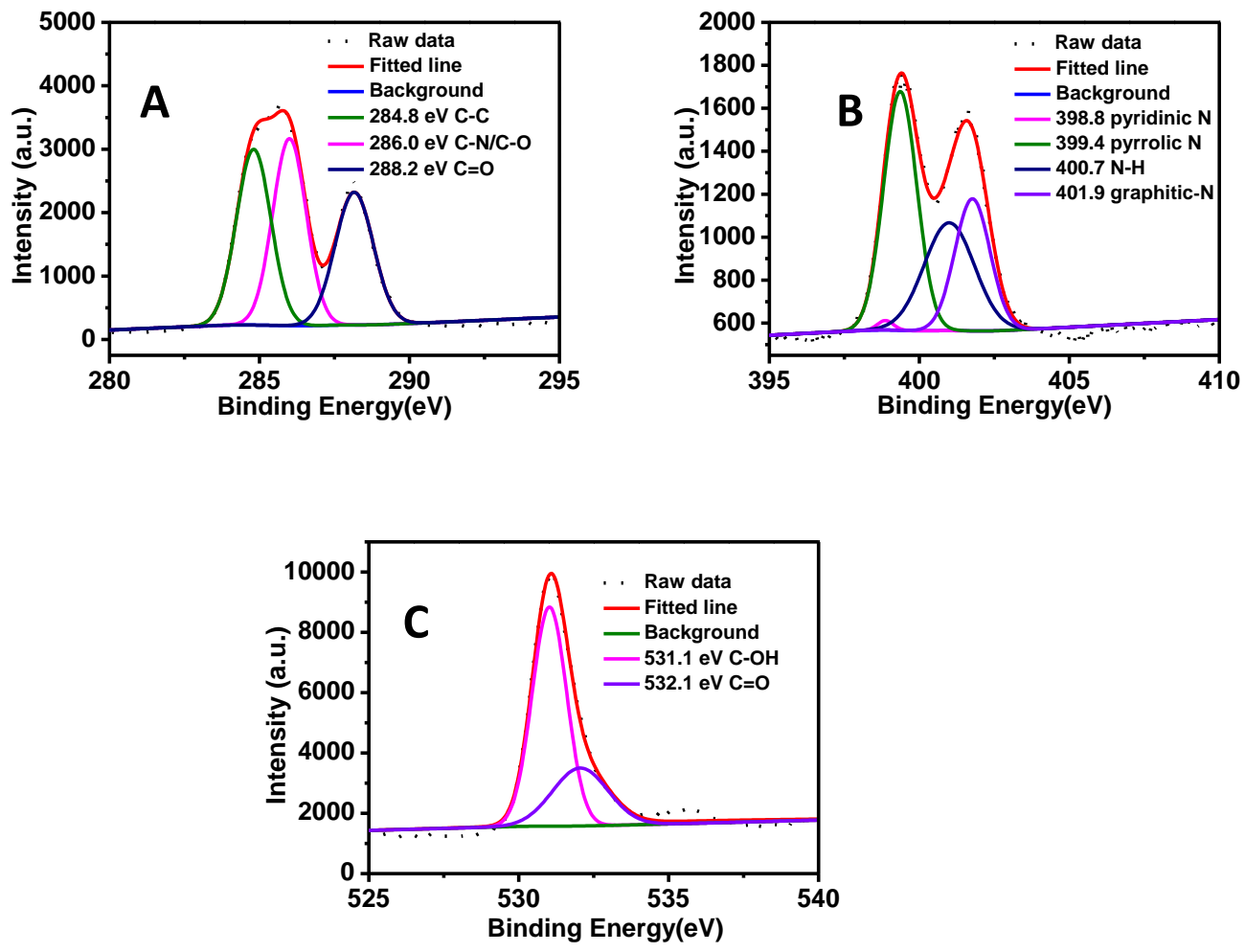

Figure S1. (A) High-resolution C1s XPS. (B) High-resolution N1s XPS. (C) High-resolution O1s XPS spectra of the CDs.

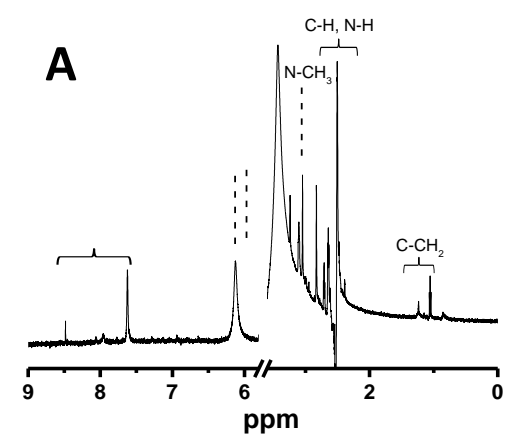

B

Figure S2. The ${ }^{1} \mathrm{H}-\mathrm{NMR}(\mathrm{A})$ and ${ }^{13} \mathrm{C}-\mathrm{NMR}(\mathrm{B})$ spectra of CDs. 


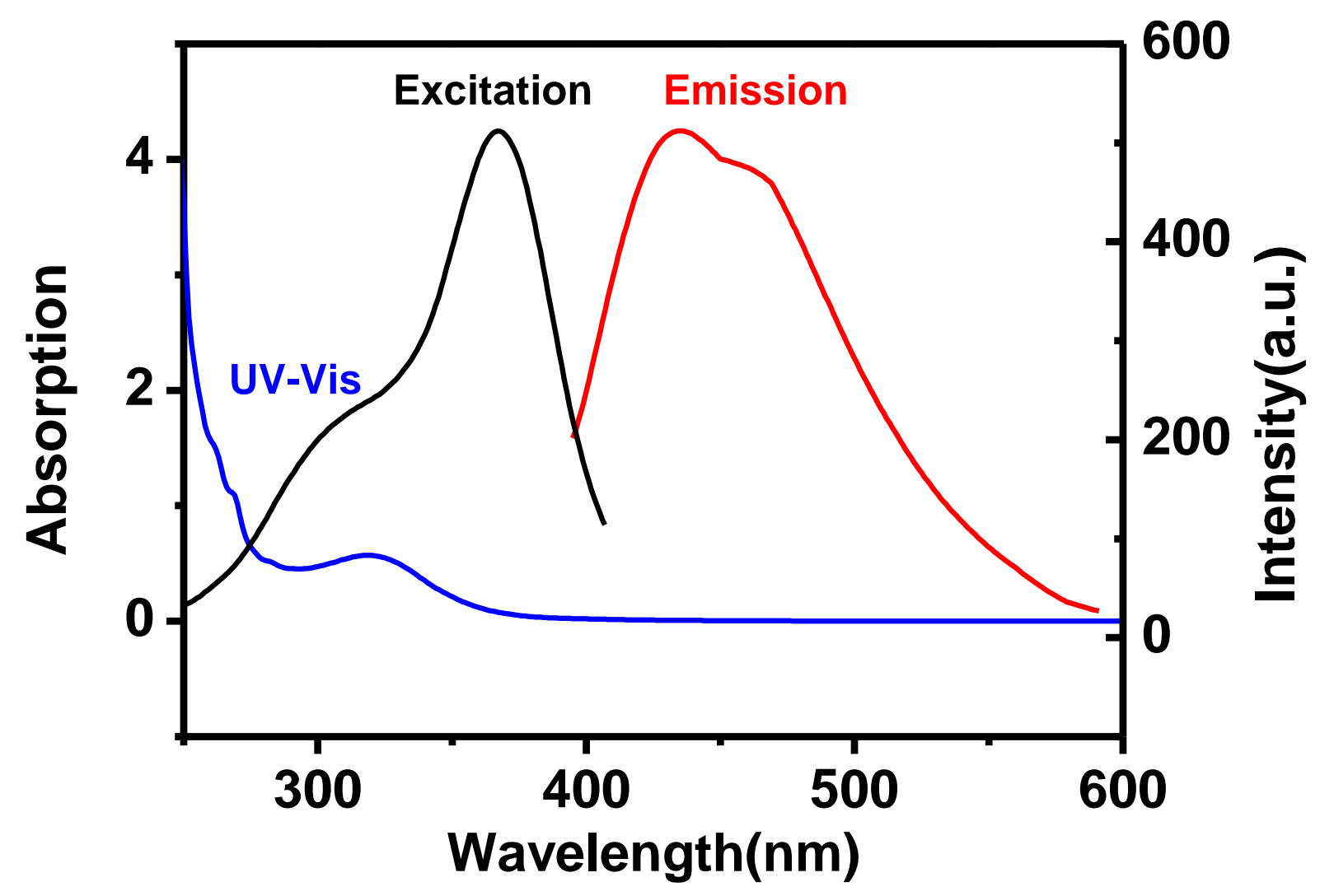

Figure S3. UV-vis absorption, excitation and emission spectra of the as-prepared CDs.

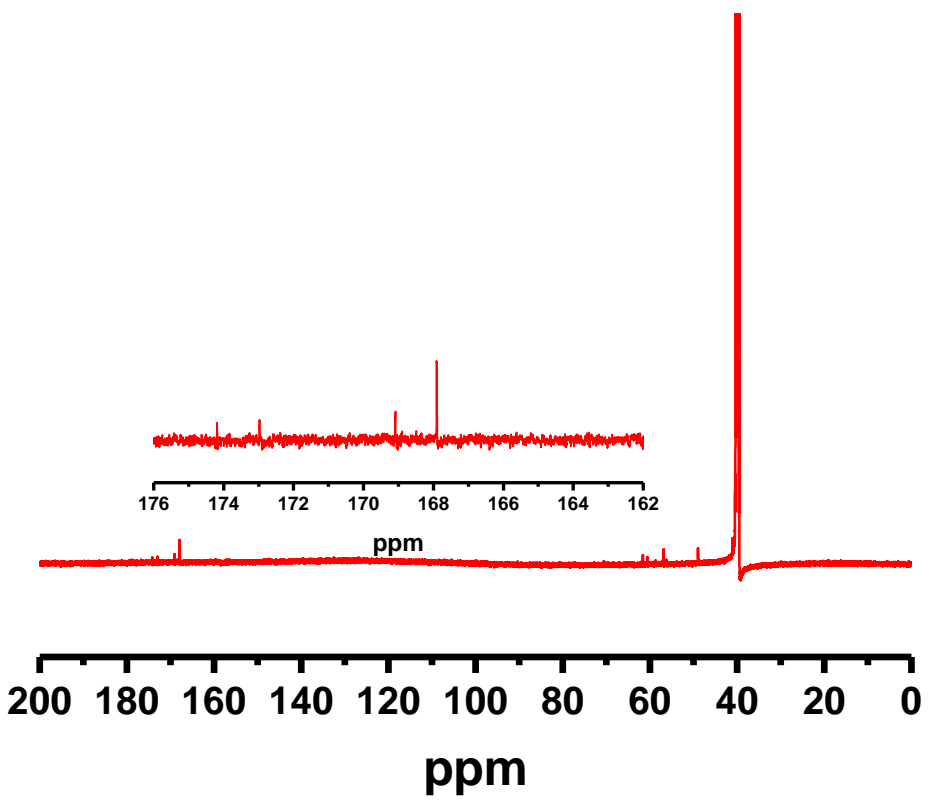

Figure S4. The ${ }^{13} \mathrm{C}-\mathrm{NMR}$ spectra of M-CDs. 


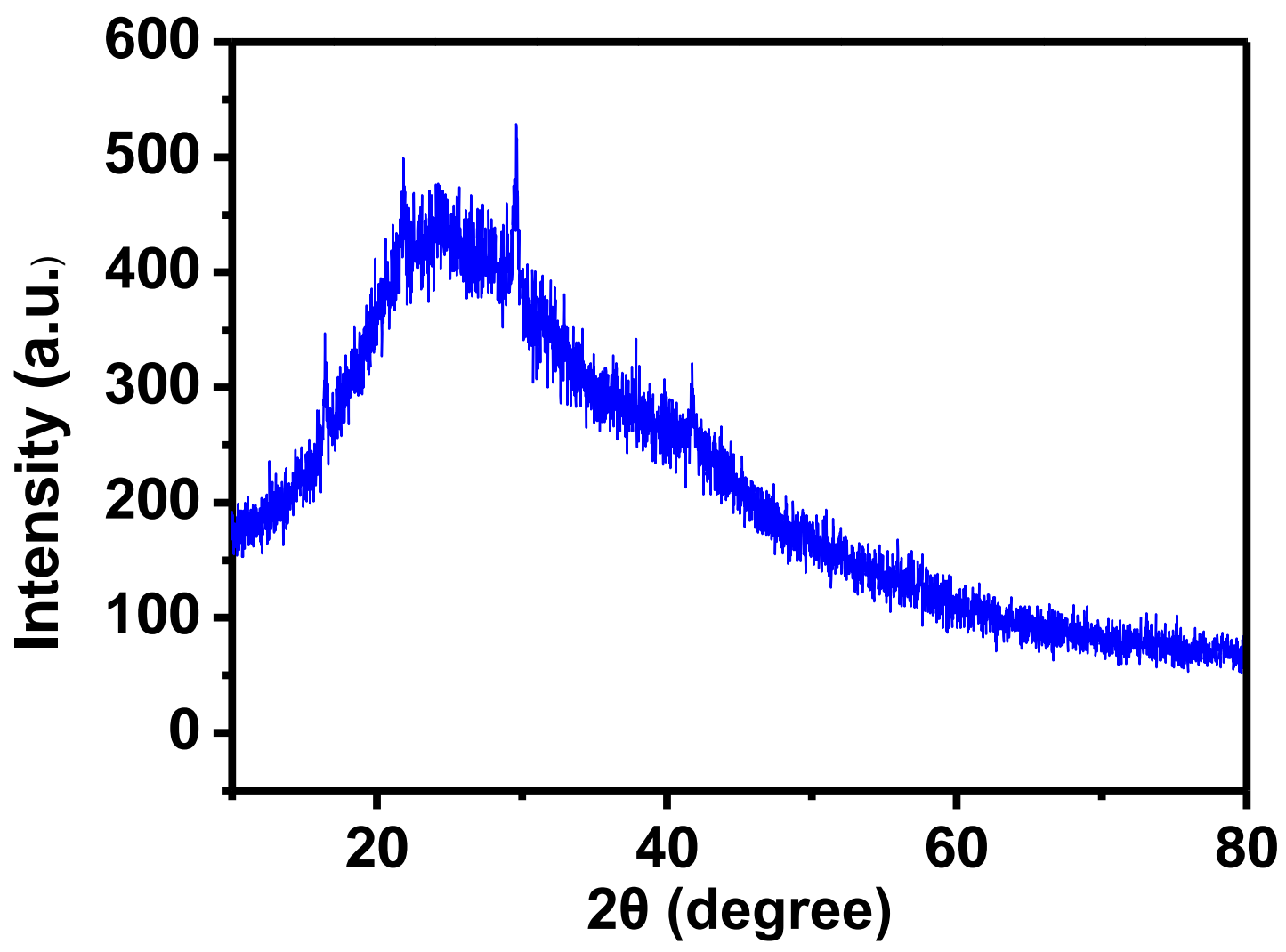

Figure S5. X-ray diffraction (XRD) pattern of the CDs.

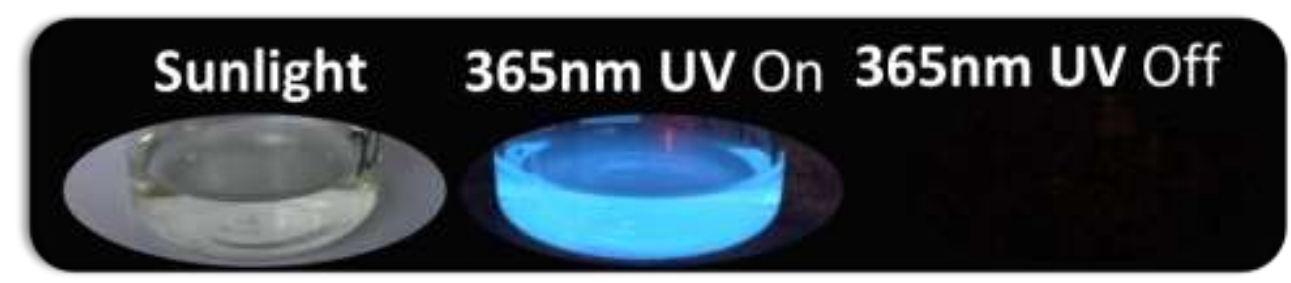

Figure S6. Photograph of CDs in aqueous solution under sunlight and 365nm UV light.

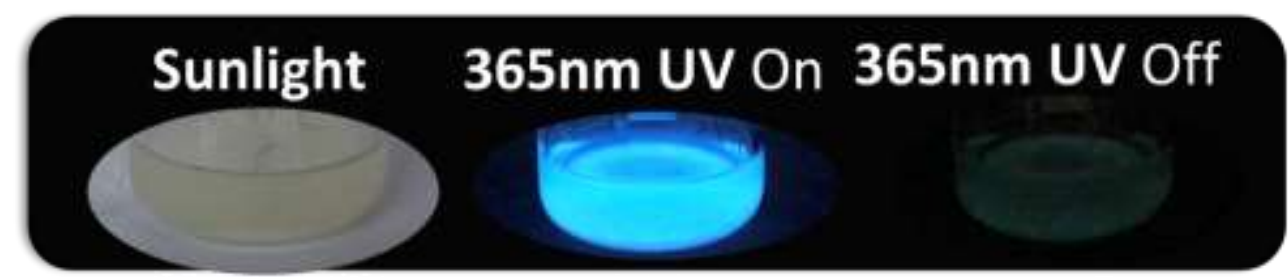

Figure S7. Photograph of M-CDs in aqueous solution under sunlight and 365nm UV light. 


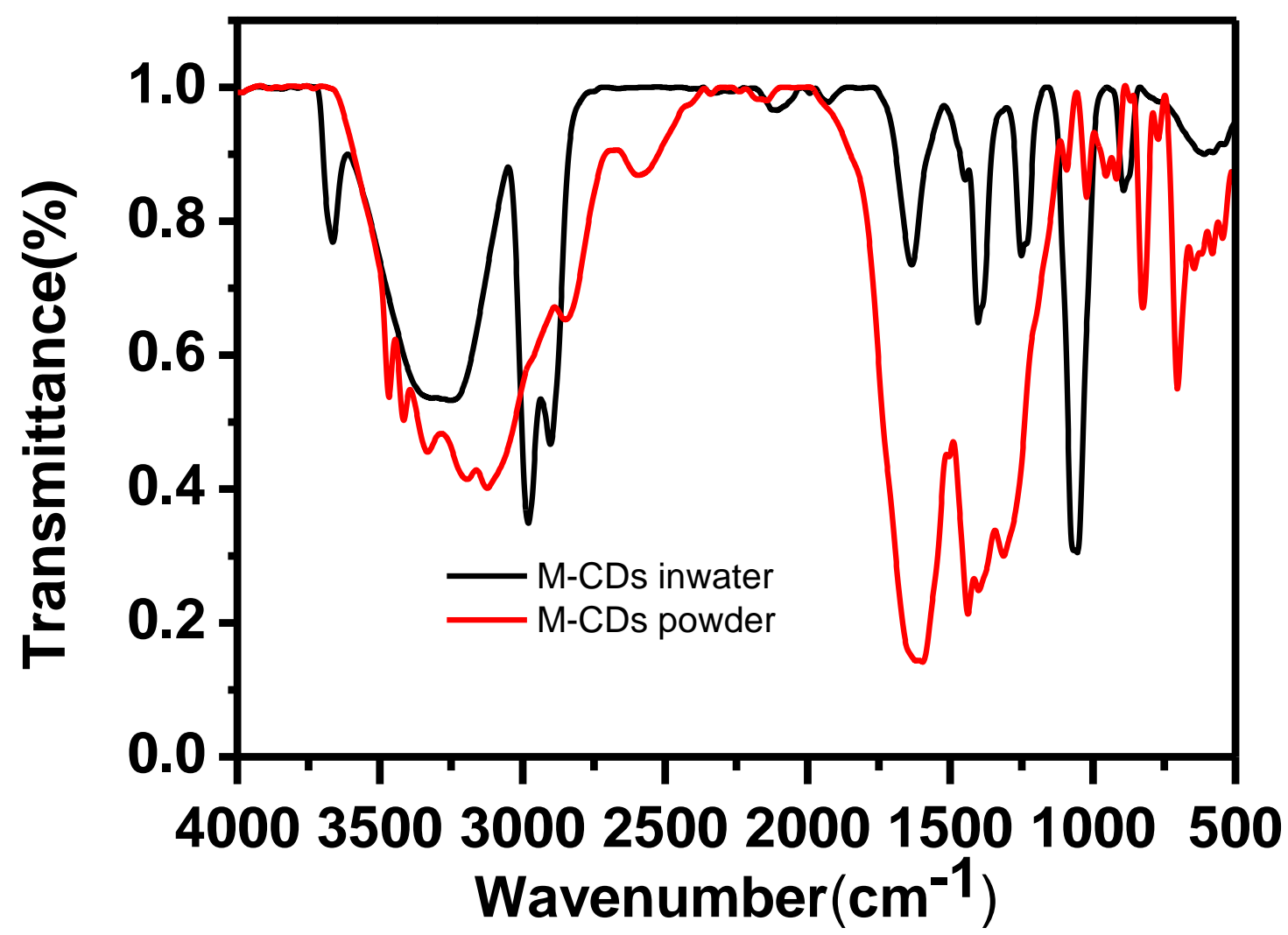

Figure S8. FT-IR spectra of M-CDs in water solution and in powder state.

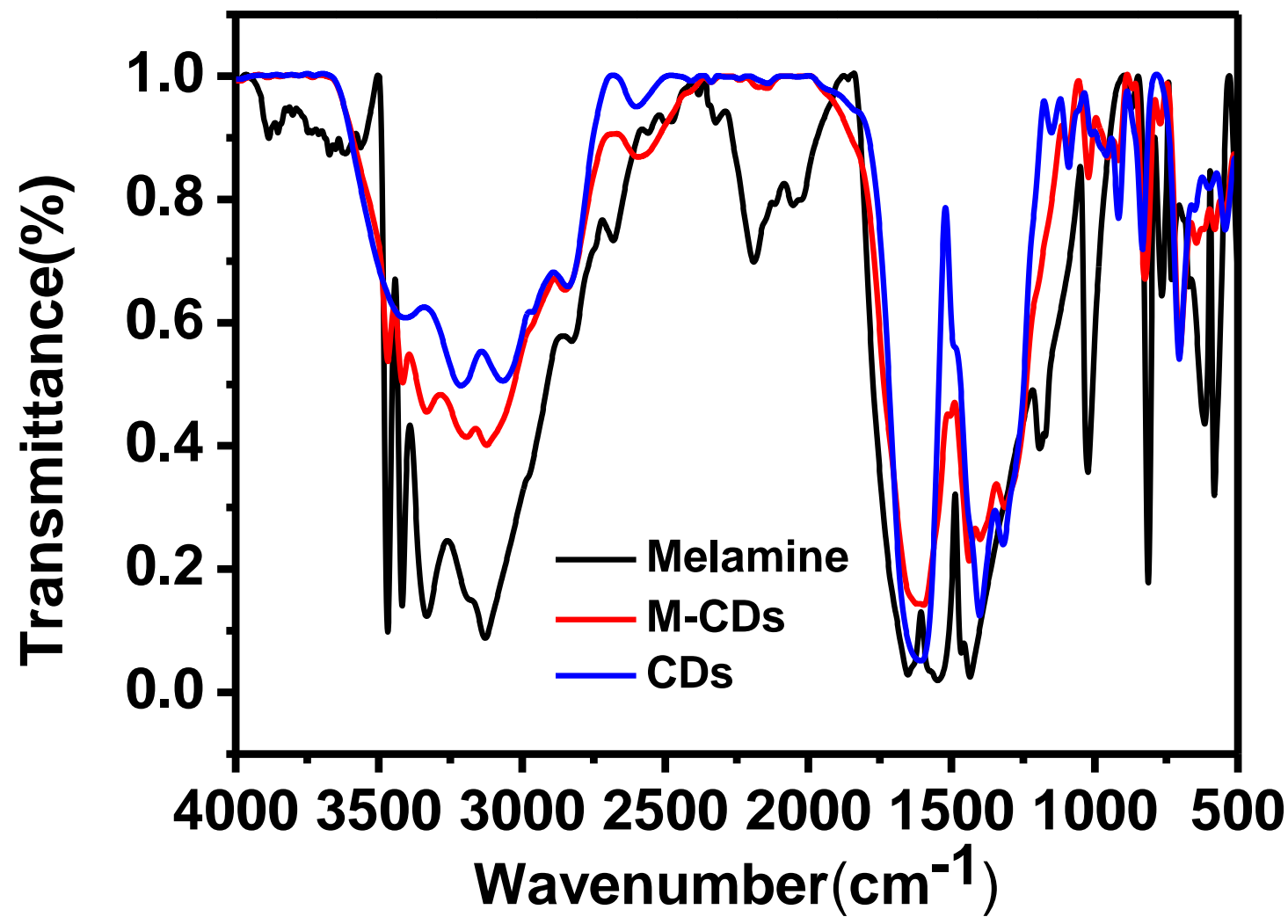

Figure S9. The FT-IR spectra of melamine, CDs and M-CDs. 


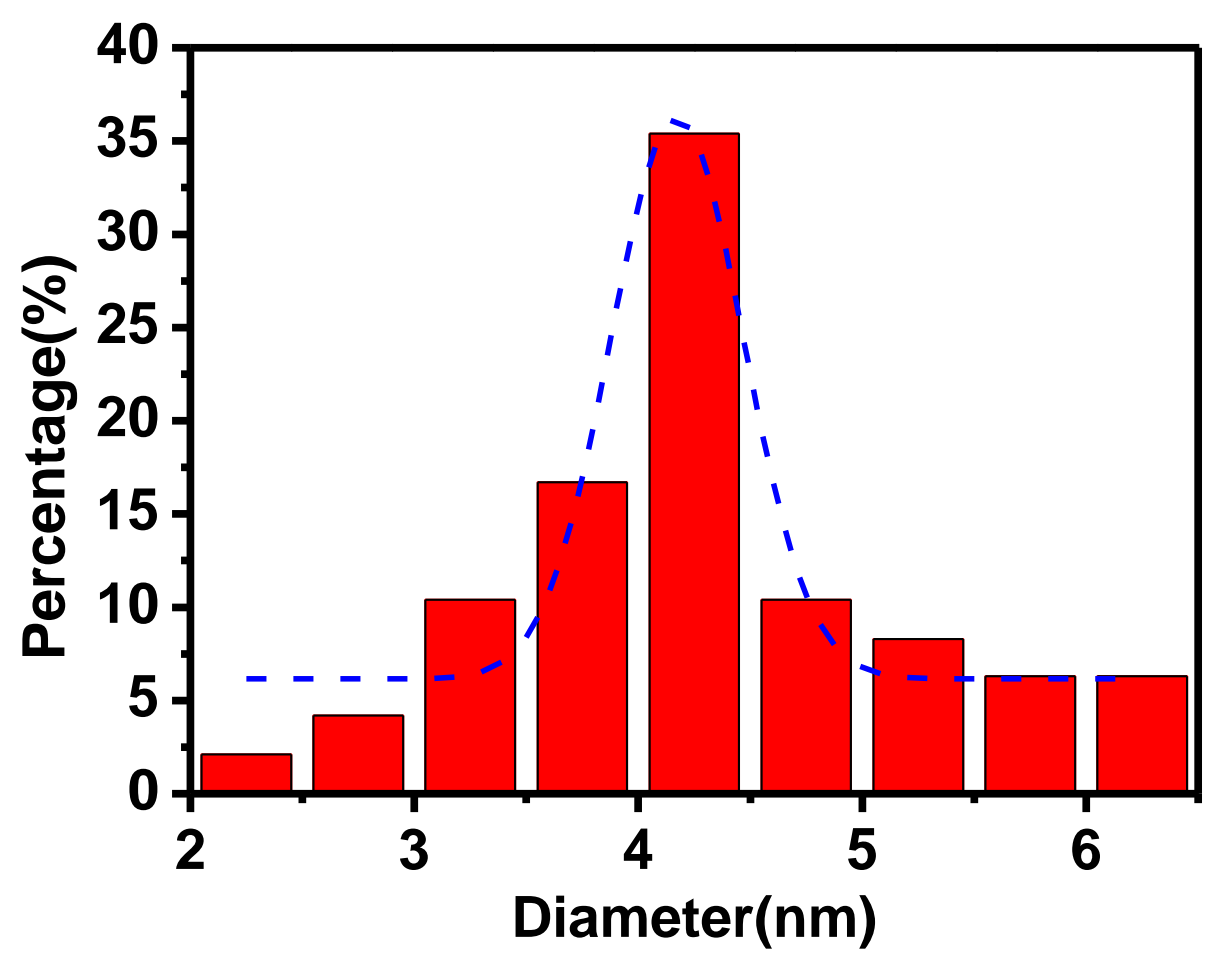

Figure S10. The size distribution of the modified single CDs.

\section{References}

1. Bai, L. Q.; Xue, N.; Wang, X. R.; Shi, W. Y.; Lu, C., Activating efficient room temperature phosphorescence of carbon dots by synergism of orderly non-noble metals and dual structural confinements. Nanoscale 2017, 9 (20).

2. Li, Q.; Zhou, M.; Yang, Q.; Wu, Q.; Shi, J.; Gong, A.; Yang, M., Efficient Room-Temperature Phosphorescence from Nitrogen-Doped Carbon Dots in Composite Matrices. Chemistry of Materials 2016, 28 (22), 8221-8227.

3. Tan, J.; Zou, R.; Zhang, J.; Li, W.; Zhang, L.; Yue, D., Large-scale synthesis of N-doped carbon quantum dots and their phosphorescence properties in a polyurethane matrix. Nanoscale 2016, 8 (8), 4742.

4. Jiang, K.; Wang, Y.; Cai, C.; Lin, H., Conversion of Carbon Dots from Fluorescence to Ultralong Room-Temperature Phosphorescence by Heating for Security Applications. Advanced Materials 2018, 30 (26), e1800783.

5. Jiang, K.; Wang, Y.; Gao, X.; Cai, C.; Lin, H., Facile, Quick, and Gram-Scale Synthesis of Ultralong Room Temperature Phosphorescent Carbon Dots by Microwave Irradiation. Angewandte Chemie International Edition 2018, $57(21)$.

6. Li, W.; Zhou, W.; Zhou, Z.; Zhang, H.; Zhang, X.; Zhuang, J.; Liu, Y.; Lei, B.; Hu, C., A Universal Strategy for Activating the Multi - Color Room - Temperature Afterglow of Carbon Dots in a Boric Acid Matrix. Angewandte Chemie 2019. 